\title{
Using focus groups to design systems science models that promote oral health equity
}

\author{
Susan S. Kum ${ }^{1,2}$, Mary E. Northridge $3,4^{*}$ (D) and Sara S. Metcalf ${ }^{1}$
}

\begin{abstract}
Background: While the US population overall has experienced improvements in oral health over the past 60 years, oral diseases remain among the most common chronic conditions across the life course. Further, lack of access to oral health care contributes to profound and enduring oral health inequities worldwide. Vulnerable and underserved populations who commonly lack access to oral health care include racial/ethnic minority older adults living in urban environments. The aim of this study was to use a systematic approach to explicate cause and effect relationships in creating a causal map, a type of concept map in which the links between nodes represent causality or influence.
\end{abstract}

Methods: To improve our mental models of the real world and devise strategies to promote oral health equity, methods including system dynamics, agent-based modeling, geographic information science, and social network simulation have been leveraged by the research team. The practice of systems science modeling is situated amidst an ongoing modeling process of observing the real world, formulating mental models of how it works, setting decision rules to guide behavior, and from these heuristics, making decisions that in turn affect the state of the real world. Qualitative data were obtained from focus groups conducted with community-dwelling older adults who self-identify as African American, Dominican, or Puerto Rican to elicit their lived experiences in accessing oral health care in their northern Manhattan neighborhoods.

Results: The findings of this study support the multi-dimensional and multi-level perspective of access to oral health care and affirm a theorized discrepancy in fit between available dental providers and patients. The lack of information about oral health at the community level may be compromising the use and quality of oral health care among racial/ethnic minority older adults.

Conclusions: Well-informed community members may fill critical roles in oral health promotion, as they are viewed as highly credible sources of information and recommendations for dental providers. The next phase of this research will involve incorporating the knowledge gained from this study into simulation models that will be used to explore alternative paths toward improving oral health and health care for racial/ethnic minority older adults.

Keywords: Oral public health, Dental public health, Oral health equity, Systems science, Agent-based modeling, Qualitative analysis, Focus group analysis, Racial/ethnic minorities, Older adults, Community-based oral health care

\footnotetext{
* Correspondence: men6@nyu.edu

${ }^{3}$ Department of Epidemiology and Health Promotion, New York University College of Dentistry, 433 First Avenue, Room 726, New York, NY 10010, USA ${ }^{4}$ Department of Sociomedical Sciences, Columbia University Mailman School of Public Health, 722 West 168th Street, New York, NY 10032, USA Full list of author information is available at the end of the article
}

(c) The Author(s). 2018 Open Access This article is distributed under the terms of the Creative Commons Attribution 4.0 International License (http://creativecommons.org/licenses/by/4.0/), which permits unrestricted use, distribution, and reproduction in any medium, provided you give appropriate credit to the original author(s) and the source, provide a link to the Creative Commons license, and indicate if changes were made. The Creative Commons Public Domain Dedication waiver (http://creativecommons.org/publicdomain/zero/1.0/) applies to the data made available in this article, unless otherwise stated. 


\section{Background}

Since the end of World War II and the subsequent increase in living standards and community water fluoridation [1], the US population has benefitted from substantial reductions in tooth decay and edentulism (complete tooth loss). The declining prevalence of edentulism in the older adult population means there is greater retention of natural teeth requiring oral health care as people age [2, 3]. Prevention and treatment efforts, technological advancements in dentistry, and dental insurance have also contributed to improved oral health [4]. Yet despite these documented improvements in oral health over time, the major oral diseases-dental caries and periodontal diseases-are still among the most prevalent chronic conditions in the US population across the life span [5].

There remain critical unmet oral health needs among children and adolescents, young and working age adults, and older adults. While the public views oral health as a priority, oral health concerns are inadequately addressed by public policies, especially for older adults [6]. For instance, because the Medicare program in the United States for persons aged 65 years and older and disabled adults does not cover routine dental care, many older adults are unable to afford the necessary preventive and restorative treatments they need [7]. Racial/ethnic minority older adults are at higher risk for edentulism than the white majority population [8]. They have also been found to be less likely to report dental visits in the past year, perhaps due to language barriers, especially among the foreign-born [9]. In addition to experiencing poorer clinical measures of oral health, racial/ethnic minority older adults also report worse self-rated oral health than their white counterparts [10].

To effectively address oral health and health care inequities requires reforming public policies for how oral health services are financed and delivered [6]. There are multiple identified factors operating at different scales that contribute to individual and population oral health inequities [11]. Therefore, strategies are needed to support and advance oral health promotion and treatment initiatives, along with oral health policy changes at local, state, and national levels. These strategies include place-based programs that apply principles of geographic targeting or directed population approaches to promote oral health equity. A primary objective of these strategies is to identify gaps in dental services and population oral health needs. Toward advancing research designed to promote oral health equity, this paper presents an approach to explicating and incorporating the perceptions and knowledge of African American, Dominican, and Puerto Rican older adults related to oral health care in their neighborhoods.
Several conceptual models for studying access to health services have been proposed. For instance, a framework devised by Penchansky and Thomas defines access as the fit between service providers and service users [12]. There are 5 dimensions for which fit is compared: (1) accessibility, i.e., the locations of service providers versus the locations of service users; (2) availability, i.e., the volume and type (supply) of services versus the volume and types of needs; (3) affordability, i.e., the cost of services versus the ability of service users to pay for them; (4) accommodation, i.e., the approach to provision by service providers versus the perceptions of appropriateness by service users; and (5) acceptability, i.e., service users' reactions to and expectations of service providers versus service providers' reactions to and expectations of service users [12]. Discrepancies in fit influence use of services, satisfaction of service users, and practices of service providers.

Powell offers an alternative taxonomic definition of access to health services that considers social accessibility to be comprised of 3 dimensions: affordability, accommodation, and acceptability [13]. In the present analyses, the question asked was: "Why do older adults go to a dental provider?" Responses were used to determine aspects of dental providers and oral health care settings that influence older adults' decisions about where they seek oral health care, i.e., social accessibility.

\section{Methods}

\section{Qualitative methods}

Valuable information for understanding and addressing problems is often held in qualitative forms of data, such as in mental models and written texts [14]. Similarly, the humanistic approach emphasizes the lived experiences and personal histories of individuals [15]. Narratives may provide meaning and context to an individual's experiences with illness and recovery [16]. Interpreting narratives may reveal characteristics associated with an individual's health status that cannot otherwise be detected, such as hope, despair, fear, guilt, and grief. Narratives may point to new hypotheses and stimulate more patient-centered research [17].

\section{Focus group approach and participants for the study}

Focus groups were conducted with a sample of 194 racial/ethnic minority men and women aged 50 years and older living in northern Manhattan who participated in one of 24 focus group sessions about improving oral health for older adults [18]. The investigators of the study selected focus groups over individual interviews because group discussions may facilitate greater disclosure by participants through reciprocity, i.e., disclosure by one participant may prompt greater disclosure by others [19]. Further, focus groups allow participants to respond 
to and elaborate on topics raised by fellow participants, thus facilitating discussion of a greater breadth of topics [19]. Finally, focus groups may be less fatiguing than individual interviews, which may be particularly important when interviewing older adults [20].

Focus group participants had to meet the following criteria: (1) aged 50 years or older; (2) attended a senior center or other community locale where older adults gather in northern Manhattan; (3) speak fluent English or Spanish; and (4) self-identify as African American, Dominican, or Puerto Rican. The demographic characteristics of the focus group participants overall and by gender are presented in Table 1.

\section{Recruitment procedure, sampling strategy, and context}

Field recruitment staff visited senior centers in northern Manhattan and directly approached older adults to explain the study, screen them for eligibility, and solicit participation in the focus groups. Senior centers were selected rather than places where older adults receive dental care in order to obtain a sample of individuals who did not necessarily have access to, or seek, dental care.

To ensure geographic and demographic representation of northern Manhattan, approximately equal numbers of participants were recruited from senior centers in each of three northern Manhattan neighborhoods: Central / West Harlem (home to large numbers of African Americans), Washington Heights
/ Inwood (home to large numbers of Dominicans), and East Harlem (home to large numbers of Puerto Ricans). These 3 neighborhoods have historically been considered as racial/ethnic enclaves, with large numbers of recent immigrants and many residents qualifying for Medicaid and other forms of public assistance. Further details of the recruitment and screening procedures are available elsewhere [18].

The study design of 24 focus groups was selected a priori in order to obtain multiple groups of each demographic segment, thereby allowing conclusions about each demographic segment to be based on multiple focus group discussions rather than on a single focus group discussion. Consistent with standard focus group techniques [21], the groups were segmented based on important characteristics that may influence the issues discussed or the ability of the members to build rapport. A total of 24 focus groups were conducted, including 12 groups of men and 12 groups of women. Within each gender set, 4 groups were conducted with African Americans, 4 groups were conducted with Dominicans, and 4 groups were conducted with Puerto Ricans. Within each gender / ethnic / racial set, half of the groups were conducted with participants who had visited a dentist in the past year and half were conducted with participants who had not visited a dentist in the past year. Ten groups were conducted in English (including two groups with

Table 1 Characteristics of participants in focus groups for the total sample and by gender, New York, NY, 2013-2015

\begin{tabular}{|c|c|c|c|c|}
\hline \multicolumn{2}{|c|}{ Participants and Focus Groups } & Total Sample & Women & Men \\
\hline \multicolumn{2}{|l|}{ Participants } & $N=194$ & $n=104$ & $n=90$ \\
\hline \multicolumn{2}{|l|}{ Focus groups } & $N=24$ & $\mathrm{n}=12$ & $n=12$ \\
\hline \multicolumn{2}{|l|}{ Characteristics } & $\%(n)$ & $\%(n)$ & $\%(n)$ \\
\hline \multirow[t]{5}{*}{ Age group in years } & $50-59$ & $14.4 \%(28)$ & $16.3 \%(17)$ & $12.2 \%(11)$ \\
\hline & $60-69$ & $34.0 \%(66)$ & $32.7 \%(34)$ & $35.6 \%(32)$ \\
\hline & $70-79$ & $36.1 \%(70)$ & $34.6 \%(36)$ & $37.8 \%(34)$ \\
\hline & $80-89$ & $11.9 \%(23)$ & $11.5 \%(12)$ & $12.2 \%(11)$ \\
\hline & $90+$ & $3.6 \%(7)$ & $4.8 \%(5)$ & $2.2 \%(2)$ \\
\hline \multirow[t]{3}{*}{ Race/ethnicity } & Dominican & $35.6 \%(69)$ & $33.7 \%(35)$ & $37.8 \%(34)$ \\
\hline & Puerto Rican & $27.3 \%(53)$ & $27.9 \%(29)$ & $26.7 \%(24)$ \\
\hline & African American & $37.1 \%(72)$ & $38.5 \%(40)$ & $35.6 \%(32)$ \\
\hline \multirow[t]{3}{*}{ Last dental visit } & Within past year & $54.1 \%(105)$ & $52.9 \%(55)$ & $55.6 \%(50)$ \\
\hline & $1-3$ years ago & $27.3 \%(53)$ & $31.7 \%(33)$ & $22.2 \%(20)$ \\
\hline & $>3$ years ago & $18.6 \%(36)$ & $15.4 \%(16)$ & $22.2 \%(20)$ \\
\hline \multirow[t]{3}{*}{ Primary language } & English & $42.3 \%(82)$ & $46.2 \%(48)$ & $37.8 \%(34)$ \\
\hline & Spanish & $48.5 \%(94)$ & $45.2 \%(47)$ & $52.2 \%(47)$ \\
\hline & Both & $9.3 \%(18)$ & $8.7 \%$ (9) & $10.0 \%(9)$ \\
\hline
\end{tabular}

Women and men did not differ significantly on any of the characteristics listed above, in accordance with the sampling strategy 
Puerto Ricans who preferred to speak English) and 14 groups were conducted in Spanish. An average of 8 older adults participated in each of the 24 focus groups.

\section{Systems science methods}

Paina and Peters propose viewing health systems through the lens of complex adaptive systems to offer insights and inform planning, implementing, monitoring, and evaluating more effective, equitable, sustainable, and context-relevant approaches to population health needs and demands [22]. For the study of complex phenomena, systems science provides a variety of research methods that are complementary to qualitative approaches [23]. These include system dynamics, agent-based modeling, social network analysis, and geographic information science [24-28].

Among the findings of a recent systematic review is that soft systems modeling techniques are likely to be the most useful addition to public health [29]. This is because the methodological positioning and subsequent metaphors in systems science, such as feedback, accumulation, and endogenous behavior, provide a way to conceptualize complex and politically sensitive problems and policies of the health system, and in the process, facilitate knowledge transfer among researchers, practitioners, and policymakers [29]. On the other hand, there also needs to be greater accountability in hard systems modeling, especially in terms of explicating the procedures used to build computer models. This would allow for other researchers and practitioners to contribute to the modeling process and assess whether or not the right questions are being addressed. More realistic, data-intensive computer models may lack transparency and flexibility, becoming too complicated and difficult to comprehend to be of practical use [30, 31].

A causal map is a visual artifact used in the field of system dynamics, a scientific approach that involves representing, testing, and modifying assumptions about dynamically complex problems attributed to feedback loops and delays [32]. Causal mapping is a method for articulating a plausible explanation for these dynamically complex problems that is used to identify feedback loops. Causal maps are integral elements in a preliminary blueprint for computer models, and in this research, are considered to be boundary objects [33]. Black defines a boundary object as "a representation-perhaps a diagram, sketch, sparse text, or prototype-that helps individuals collaborate effectively across some boundary, often a difference in knowledge, training, or objective." ([34], 76, p).

\section{Integrating qualitative and systems science methods in the data analysis}

Importantly, a causal map depicts cause-and-effect relationships, where arrows between variables are used to specify the direction of cause and effect. A polarity is assigned to each relationship indicating whether the effect (variable Y) increases or decreases relative to the cause (variable $\mathrm{X}$ ) ceteris paribus, i.e., holding all else equal.

A protocol proposed by Kim and Andersen for coding qualitative text data and developing causal maps from purposive text data was adapted here to analyze decisions on where older adults seek oral health care [14]. The initial stage involves critically evaluating the text to extract data segments and cause-and-effect relationships. This corresponds to open coding, i.e., the summarizing and division of text that captures specific phenomena or experiences that may be used as codes. As codes are grouped, dominant patterns or themes emerge and are observed and subsequently coded. The next stage involves the grouping together of similar data segments to arrive at more generalized cause-and-effect relationships and implicit structures in order to construct a composite causal map. This step corresponds to axial coding, i.e., the organization of relationships among categories of codes to establish relationships between categories. Implicit structures, i.e., intermediate variables, guide the merging of categories of codes.

A topic guide consisting of open-ended questions was developed by the research team and used by the focus group moderators to facilitate the sharing of personal experiences among participants (see Additional file 1). The initial categories for organizing the qualitative data were based upon the topic guide; additional categories were included during subsequent readings of the transcripts. Focus groups with content related to categories of interest were indicated and recorded. An iterative process was used to extract excerpts of transcript text, which are referred to as data segments.

Data segments are typically a single response from a participant, i.e., a portion of text indicated in the focus group by "P:" indicating "Participant:" in the transcripts. For certain data segments, it was important to capture the dialogue. Hence, a forward slash (/) is used to indicate multiple responses. Cause-and-effect relationships are then identified from data segments.

Table 2 presents the topics by type (either finding dental providers or going to dental providers) identified from the 24 focus groups.

Topics refer to the pattern or theme that was determined by examining a grouping of similar data segments and were identified at the time of extraction of data segments. Labels were then assigned to types and topics to facilitate the filtering of the data segments in a spreadsheet. Next, each data segment was assigned a data segment Type, Topic, and identification number (SegmentID). When assigning SegmentIDs, replicate 
Table 2 Decisions on where to go for oral health care organized by types and topics

\begin{tabular}{ll}
\hline Types (in shaded rows) and Topics & Label \\
\hline Finding dental providers & finding \\
Information from insurance company & insurance \\
Third-party resources (1-800-Dentist, 311, Internet) & third-party \\
Referral from another health provider & referral \\
Recommendations from friends, family, community & recommendation \\
Going to dental providers & going \\
Recommendations from friends, family, community & recommendation \\
Dental care environment & environment \\
Training and credentials (title) of oral health providers & credentials \\
Character of oral health providers & character \\
Shared language or culture and understanding of community & understanding \\
(Dis)Trust in oral health provider & trust \\
Quality of dental care & quality \\
Dental treatment options & treatment \\
Dental need (urgent care) & need \\
Appointments & appointments \\
Insurance coverage and cost of care & cost \\
\hline
\end{tabular}

entries were removed that had been introduced erroneously during data extraction.

\section{Results}

Figure 1 provides an illustration of the process by which a data segment and corresponding focus group segmentation information is recorded and organized.

Certain data segments yielded more than a single cause-and-effect relationship; thus, there are 287 relationships from 240 data segments. The data segment shown in Fig. 1a is SegmentID 56, which was extracted from focus group 5, comprised of Dominican men who had received a dental visit in the past year (translated into English from Spanish). This data segment was used to explicate 2 cause-and-effect relationships: the first for finding dental providers and the second for going to dental providers. This is an especially interesting data segment because it represents both the view of the speaker and the view of his friend.

A data segment cause (dsCause) and a data segment effect (dsEffect) were then identified for each data segment. Attempts were made to extract terms from each data segment to express dsCause and dsEffect. To continue with the illustration, Fig. $1 \mathrm{~b}$ presents the cause-and-effect relationships identified for SegmentID 56. For data segments where it was difficult to maintain complete data integrity, terms derived from the interpretation of the first author for the data segment were used.

Similar expressions of cause and effect relationships, aided by the filtering of labels, were then generalized into a causal map cause (cmCause) and a causal map effect (cmEffect). For example, Fig. 1c presents the cmCause and cmEffect to which SegmentID 56 contributed. Data segments in the same topic group were compared to derive a term that was representative of the relationships expressed in that subset of data segments. Deliberate attempts were made to use variables that were already assigned; new variables were typically generated to clarify relationships. Each of the generalized relationships was then assembled in Vensim software [35] to provide a visual overview of the existing relationships. Certain of the generalized relationships were collapsed during the process of assembly and intermediate structures were added. The order in which the variables were assigned a cmID, indicating the generalized cause and effect relationships, followed the descending order of topics presented in Table 1. One of the generalized relationships in a topic group would often provide a link to another topic group. To complete the example, Fig. 1d presents an illustration of how the generalized cause-and-effect relationships are summarized and tabulated.

The behavior (noted simply as Behavior) of the cmCause and cmEffect was then assessed. The Behavior was articulated to check the logic of each cause and effect relationship and assign a polarity to that relationship. The polarity of the relationship, that is, whether the behavior of the cause and effect changes in the same direction (positive) or in the opposite direction (negative), is recorded in Relationship Type. For a positive relationship type, an increase (decrease) of the cause leads to an increase (decrease) of the effect; for a negative relationship type, an increase (decrease) of the cause leads 
a Data segment is identified and extracted

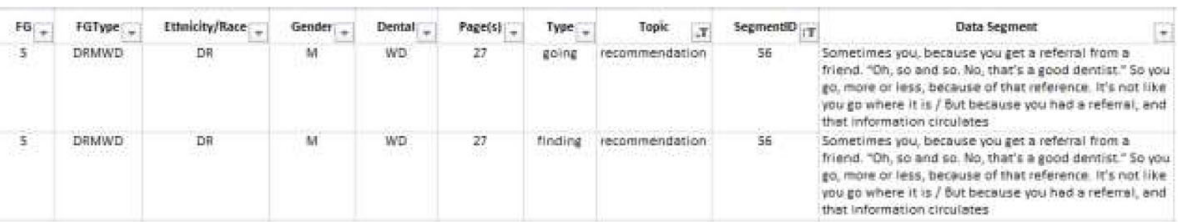

b Cause-and-effect relationships are explicated

\begin{tabular}{|c|c|c|c|c|}
\hline SegmentID & Data Segment & dsCause & dsEffect & $\mathrm{cmlD}$ \\
\hline 56 & $\begin{array}{l}\text { Sometimes you, because you get a referral from a } \\
\text { friend. "Oh, so and so. No, that's a good dentist." So you } \\
\text { go, more or less, because of that reference. It's not like } \\
\text { you go where it is / But because you had a referral, and } \\
\text { that information circulates }\end{array}$ & $\begin{array}{l}\text { satisfaction with } \\
\text { dental provider }\end{array}$ & recommendation & 7 \\
\hline 56 & $\begin{array}{l}\text { Sometimes you, because you get a referral from a } \\
\text { friend. "Oh, so and so. No, that's a good dentist." So you } \\
\text { go, more or less, because of that reference. It's not like } \\
\text { you go where it is / But because you had a referral, and } \\
\text { that information circulates }\end{array}$ & $\begin{array}{l}\text { reference from } \\
\text { friends }\end{array}$ & $\begin{array}{l}\text { knowledge about } \\
\text { dental providers }\end{array}$ & 6 \\
\hline
\end{tabular}

C Similar expressions of cause and effect relationships contribute to a generalized causeeffect relationship

\begin{tabular}{|c|c|c|c|c|}
\hline cmlD & cmCause & cmEffect & Behavior & Relationship Type \\
\hline 7 & $\begin{array}{c}\text { satisfaction with } \\
\text { dental provider }\end{array}$ & $\begin{array}{c}\text { dental provider } \\
\text { recommendations }\end{array}$ & $\begin{array}{c}\text { great satisfaction with dental provider, } \\
\text { more dental provider recommendation }\end{array}$ & positive \\
\hline 6 & $\begin{array}{c}\text { communication } \\
\text { about dental } \\
\text { experience }\end{array}$ & $\begin{array}{c}\text { dental provider } \\
\text { recommendations }\end{array}$ & $\begin{array}{c}\text { more communication about dental } \\
\text { experience, more dental provider } \\
\text { recommendations }\end{array}$ & positive \\
\hline
\end{tabular}

\section{d Generalized cause-and-effect relationships are summarized and tabulated}

\begin{tabular}{|cccccc|}
\hline cmID & cmCause & cmEffect & Relationship & $\begin{array}{c}\text { Data } \\
\text { Segments }\end{array}$ & $\begin{array}{c}\text { Focus } \\
\text { Groups }\end{array}$ \\
\hline 1 & resources from insurance plan & knowledge about dental providers & positive & 11 & 8 \\
2 & third-party services & knowledge about dental providers & positive & 4 & 4 \\
3 & computer literacy & knowledge about dental providers & positive & 2 & 2 \\
4 & referrals from health providers & knowledge about dental providers & positive & 9 & 8 \\
5 & dental provider recommendations & knowledge about dental providers & positive & 9 & 7 \\
\hline 6 & communication about dental experience & dental provider recommendations & positive & 25 & 14 \\
\hline \hline 7 & satisfaction with dental provider & dental provider recommendations & positive & 9 & 5 \\
\hline 8 & dental provider recommendations & expectations of quality dental care & positive & 1 & 1 \\
\hline
\end{tabular}

Fig. 1 Process by which a data segment is recorded and organized. An illustration of the process by which an extracted data segment is recorded and organized. Specifically, data segment 56 (SegmentID 56) originates from focus group 5 conducted with Dominican men who received oral health care in the past year (translated into English from Spanish). The 4 panels correspond to the following steps: (a) Data segment is identified and extracted; (b) Cause and effect relationships are explicated; (c) Similar expressions of cause and effect relationships contribute to a generalized cause-and-effect relationship; and (d) Generalized cause-and-effect relationships are summarized and tabulated

to a decrease (increase) of the effect. A causal map ID (cmID) was assigned after all of the 37 generalized cause-and-effect relationships were established. To complete the illustration, a summary and tabulation of the generalized cause-and-effect relationships used to systematically construct the causal map is presented in Fig. 1d, with the contribution of SegmentID 56 highlighted in yellow.

Modifications to the Kim and Andersen protocol [14] for constructing a causal map were motivated by the need to be able to efficiently compare different topics and data segments. Instead of attempting to capture all of the attendant details, data segments were aggregated to highlight the major constructs and the potential mechanisms that connect these constructs. Both the task of manually extracting and organizing the text and the task of generalizing the selected text were time intensive. Yet it was only through multiple and iterative readings of the text that the analyses were effectively framed. This was especially important in generating the causal map, since justifying the underlying logic of relationships required considerable abstraction. 
Finally, a composite causal map representing the experience of the patient was systematically constructed to explicate the generalized relationships involved in the decision-making process of dental provider choice (Fig. 2).

These considerations correspond to the access dimensions of acceptability, accommodation, and affordability, i.e., social accessibility to oral health care. Two separate but related issues are manifest: the first involves finding dental providers, knowledge about dental providers, and the places where dental providers practice; and the second involves going to and returning to dental providers, more specifically, the circumstances at dental practices that motivate dental visits.

The focal variable of the causal map is dental visits (highlighted in orange), an indicator of utilization of or realized access to the oral health care system. The two issues of finding and going to dentists are represented, respectively, as knowledge about dental provider and motivation for dental visit (also highlighted in orange in the causal map).
The first column in Table 3 lists the 19 variables in the causal map that are included in any of 12 feedback loops involved in finding or going to a dental provider.

All 12 feedback loops include the focal variable dental visits. Three feedback loops are associated with finding dentists - knowledge about dental provider - and 9 feedback loops are associated with going to dentists motivation for dental visit. Variables that are not included in any feedback loops are listed in the last column of Table 2 as preceding variable(s), i.e., a cause of the associated variable in the feedback loop.

Below illustrative data segments are presented and discussed, including those related to the example in Fig. 1 (SegmentID 56). Participant responses for how they found dental providers included: resources from insurance plans; phone calls to third-party services; Internet searches; referrals from health care providers; and recommendations from relatives and friends. For example, an African American man explained how he was referred by a dental provider to another dental provider

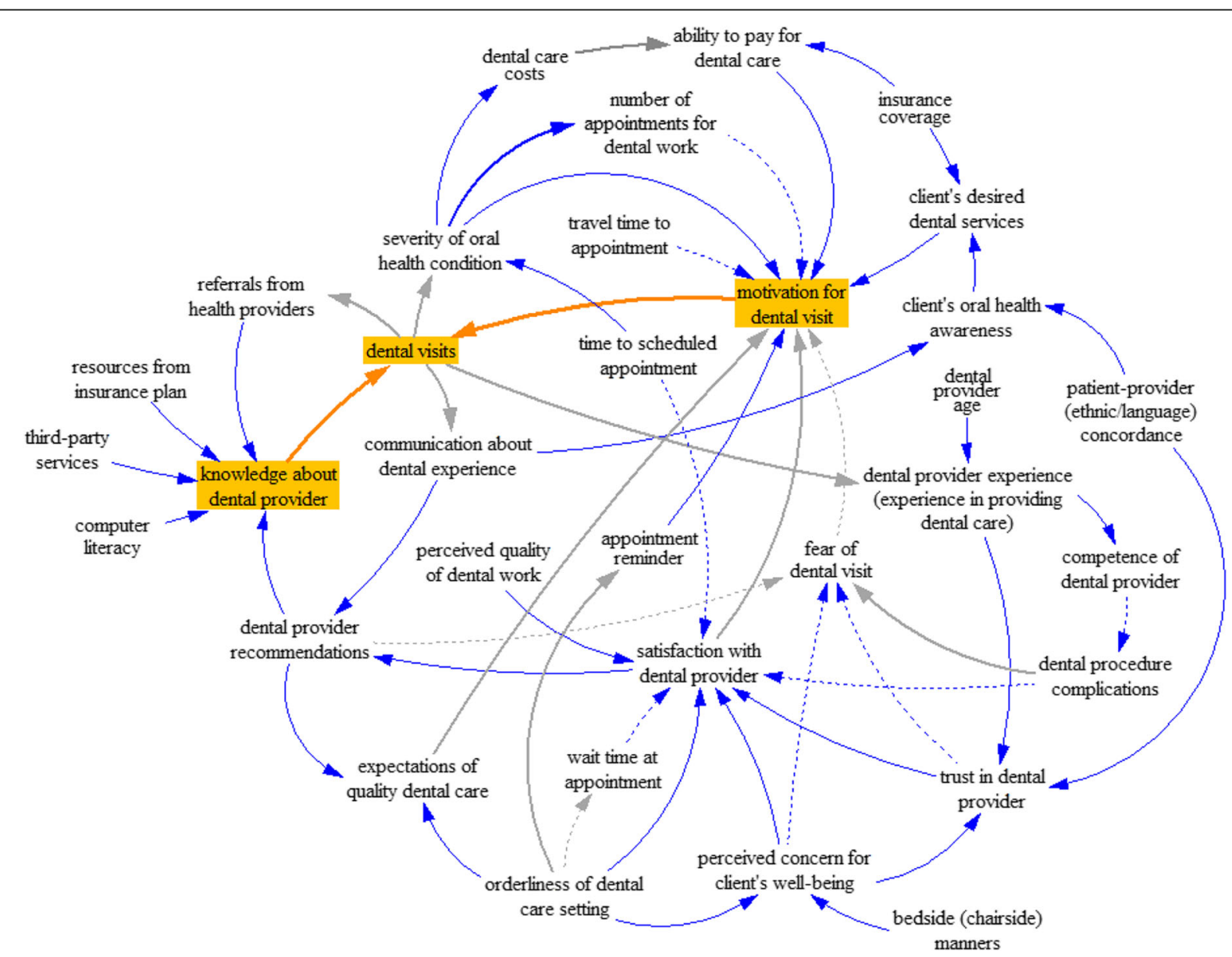

Fig. 2 Causal map derived from focus group data. A composite causal map of decisions on where to go for oral health care based on information extracted from focus groups with African American, Dominican, and Puerto Rican older adults. The solid arrows indicate a positive effect (same direction), whereas the dashed arrows indicate a negative effect (opposite direction) 
Table 3 Feedback loops of decisions on where to go for oral health care

\begin{tabular}{|c|c|c|c|c|c|c|c|c|c|c|c|}
\hline \multirow{2}{*}{ Variable in Feedback Loop } & \multicolumn{2}{|c|}{ Finding } & \multicolumn{8}{|c|}{ Going } & \multirow{2}{*}{$\begin{array}{l}\text { Preceding Variable(s) } \\
\mathrm{p}^{*}=\text { perceived }\end{array}$} \\
\hline & 1 & 2 & 4 & 45 & 6 & 7 & 8 & 9 & 10 & \begin{tabular}{l|l}
11 & 12 \\
\end{tabular} & \\
\hline \multicolumn{12}{|l|}{ dental visits } \\
\hline $\begin{array}{l}\text { referrals from } \\
\text { health providers }\end{array}$ & & & & & & & & & & & \\
\hline $\begin{array}{l}\text { communication about } \\
\text { dental experience }\end{array}$ & & & & & & & & & & & \\
\hline $\begin{array}{l}\text { client's oral health } \\
\text { awareness }\end{array}$ & & & & & & & & & & & patient-provider concordance \\
\hline $\begin{array}{l}\text { client's desired } \\
\text { dental services }\end{array}$ & & & & & & & & & & & insurance coverage \\
\hline $\begin{array}{l}\text { dental provider experience } \\
\text { (experience providing dental care) }\end{array}$ & & & & & & & & & & & dental provider age \\
\hline $\begin{array}{l}\text { trust in } \\
\text { dental provider }\end{array}$ & & & & & & & & & & & $\begin{array}{l}\text { patient-provider concordance } \\
\mathrm{p}^{*} \text { concern for client's well-being }\end{array}$ \\
\hline $\begin{array}{l}\text { competence of } \\
\text { dental provider }\end{array}$ & & & & & & & & & & & \\
\hline $\begin{array}{l}\text { dental procedure } \\
\text { complications }\end{array}$ & & & & & & & & & & & \\
\hline $\begin{array}{l}\text { satisfaction with } \\
\text { dental provider }\end{array}$ & & & & & & & & & & & \begin{tabular}{|l|}
$\mathrm{p}^{*}$ quality of dental work \\
$\mathrm{p}^{*}$ concern for client's well-being \\
orderliness of dental care setting \\
time to scheduled appointment \\
wait time at appointment
\end{tabular} \\
\hline \multicolumn{12}{|l|}{$\begin{array}{l}\text { dental provider } \\
\text { recommendations }\end{array}$} \\
\hline $\begin{array}{l}\text { knowledge about } \\
\text { dental provider }\end{array}$ & & & & & & & & & & & $\begin{array}{l}\text { resources from insurance plan } \\
\text { third-party services } \\
\text { computer literacy }\end{array}$ \\
\hline $\begin{array}{l}\text { expectations of } \\
\text { quality dental care }\end{array}$ & & & & & & & & & & & orderliness of dental care setting \\
\hline $\begin{array}{l}\text { fear of } \\
\text { dental visit }\end{array}$ & & & & & & & & & & & $\mathrm{p}^{*}$ concern for client's well-being \\
\hline $\begin{array}{l}\text { severity of } \\
\text { oral health condition }\end{array}$ & & & & & & & & & & & time to scheduled appointment \\
\hline $\begin{array}{l}\text { number of appointments } \\
\text { for dental work }\end{array}$ & & & & & & & & & & & \\
\hline $\begin{array}{l}\text { dental care } \\
\text { costs }\end{array}$ & & & & & & & & & & & \\
\hline $\begin{array}{l}\text { ability to pay } \\
\text { for dental care }\end{array}$ & & & & & & & & & & & insurance coverage \\
\hline $\begin{array}{l}\text { motivation for } \\
\text { dental visit }\end{array}$ & & & & & & & & & & & $\begin{array}{l}\text { travel time to appointment } \\
\text { appointment reminder }\end{array}$ \\
\hline
\end{tabular}

for specialized care: "So, finally you got a lot of dentists who are really at another level. I've been to dentist who have been like, "Listen, I can't do this, but your union is going to pay." Mr. [name] is a good dentist for, what do you call it? The root canal!" (focus group 17: African American men with a dental visit in the past year) Family members and friends were considered to be both important sources of recommendations for finding dental providers and influential in going to dental visits.

The role of referrals and recommendations in finding dentists are captured in feedback loops 1, 2, and 3; the influence of communication about dental experiences are captured in feedback loops 4,5 , and 6 . These feedback loops indicate the potential of using social networks to deliver knowledge and change attitudes and practices towards oral health care. For example, an African American man shared the following remarks: "She'll [wife] find a dentist. [pause]. She'll notice where I'm taking all the pill because it don't matter to me. I just went to bed. I won't notice [pause] things like that. But she will. She's good that way." (focus group 17: African American men with a dental visit in the past year) Older adults who lack the support of family and friends may neglect their health and encounter difficulties in obtaining resources to address their health needs, such as information about oral health care options in their neighborhoods and transportation assistance.

According to participants, information and opinions about dental providers and care settings often circulate through word of mouth among community members 
that may affect their reputations. The following quote from a Dominican man emphasizes the impact of communication in decision-making for selecting dentists:

\section{"There is something important that I want to explain here, the vast majority of us Hispanics look for our doctors through references, this is very important. In other words if a doctor is good, the neighbor will say... look, so and so is a wonderful dentist. Then we start looking for references. And it's through the references that we start communicating with one another and we even make an appointment to go see that doctor. Then, if the doctor is bad and did not complete the work well, for this and that reason. It's always like that, the neighbor will communicate with the second person and that spreads the word. In other words, a job well done will be well received by the community, but people will also know about bad work." (focus group 15: Dominican men with a dental visit in the past year, translated into English from Spanish)}

Several participants were frightened about going to the dentist either because of fear and the associated pain of dental procedures or the fear of contracting diseases at the dental office. Recommendations from trusted family members and friends may minimize such fears because dental providers and oral health care settings have already been vetted (feedback loop 6). A Dominican woman confidently stated: "I say, 'You are afraid? But let me tell you, I have a dentist you will love. Here is the phone number. Here is the phone number. Go to him and you will remember me." (focus group 14: Dominican women without a dental visit in the past year, translated into English from Spanish).

\section{Discussion}

In systems science, both the problems and their solutions are understood as being generated from within the system. Social disparities in oral health result from factors at multiple scales [11]. With regular dental hygiene and professional care, adverse oral health outcomes such as tooth loss may often be prevented. Oral health behaviors and practices are transferred through social relationships throughout the life course, which may either increase or decrease social disparities in oral health. For example, children often adopt the behaviors and practices of their parents. Social relationships among older adults provide mechanisms for the exchange of resources, such as information that is critical in decision-making.

While the life course approach is useful in understanding oral health inequities, there are empirical challenges in testing the proposed theoretical models [36, 37]. Problems with using retrospective studies include the inability to control for exposure times, selection bias due to loss to follow-up, and underrepresentation of racial/ ethnic minorities [38]. Agent-based modeling has been proposed as a way forward since it facilitates representation of multiple scales and heterogeneity $[39,40]$. Moreover, agent-based models are theory-based and data-driven, and allow for the testing of different plausible mechanisms [41].

A computer model may be used to communicate and learn about the impact of life events and social relationships on oral health. The proposed model design contributes to an existing portfolio of models that have been informed by quantitative and spatial data, as well as the experiences and expertise of research team members [33, 42-46]. Here particular insights from older adults' experiences that are reflected in focus groups transcripts are included, along with supplementary knowledge about the older adult population in Manhattan and the Bronx. The model is designed to simulate: (1) the life course, i.e., life stages and life events; (2) oral health status, oral health care seeking orientation, and oral health care use; and (3) multiple social relationships as dynamic social networks based on person agent attributes and geographic proximity.

Below are key insights gleaned from the focus groups that are considered important in the model design. First, the inability to pay for oral health care is a significant barrier to accessing services. Second, family members and friends are both important sources of recommendations for finding dental providers and influential in motivating dental visits. Third, participants believe there is a lack of information about oral health in the community and they would like more information about oral health and health care.

The design includes 2 active agent classes: person (older adult, dental provider) and place. Place is further specified into home, work, third place, e.g., senior center, public library, religious institution, and dental clinic [46]. Characteristics of all place agents will include a unique identification number and an indication of status (either open or closed). An additional characteristic of dental clinics is the types of insurance accepted. Further, the identification numbers of older adults who visited each dental clinic and the dates of these visits as well as reminder messages to older adults regarding scheduled appointments will need to be tracked. An additional characteristic of third places is whether health outreach events are held at each location, and if so, the identification number of participants at outreach events, and the participants who needed a referral to a dental clinic affiliated with the outreach events.

According to the focus group transcript analysis, availability (the supply of dental providers) and accessibility (the means of traveling to oral health care) were not 
perceived to be significant barriers to oral health care. Rather, the inability to pay for oral health care, poor relationships with dental providers, and lack of information on oral health and health care were the major challenges. Therefore, the model design includes 2 types of interventions: (1) social and behavioral interventions; and (2) policy interventions. The first set of modeled interventions would involve community-based outreach education and delivery at places in the neighborhood that provide information, and use different social relationships to direct information and influence changes in oral health care-seeking orientation and oral health care status. The second set of proposed interventions would involve health insurance coverage, specifically, the impact of expanding and ensuring dental insurance coverage throughout the life span, and reducing restrictions to preventive oral health care.

\section{Conclusions}

The findings of this study support the multi-dimensional and multi-level perspective of access to oral health care and affirm a theorized discrepancy in fit between available providers and patients. The presence of resources does not directly translate into use of services by racial/ ethnic minority older adults. Despite the relatively high volume of dental providers and the range of transportation options, focus group participants did not believe that their oral health needs were being adequately addressed, whether or not they had recently visited a dentist.

The systematic approach to explicating cause and effect relationships from focus group transcripts introduced here may prove transferable to other research contexts. The product of this approach, a causal map, provides a visual representation of major factors and relationships involved in the decision-making process.

From both epistemological and ontological standpoints, however, system dynamics involves more than the mechanics of creating a causal map. Rather, there is a philosophical understanding that in order to solve large, complex problems, it is important and effective to consider the needs of others [47]. The ability to incorporate qualitative data into a causal map allows direct inclusion of the views of underrepresented populations into the hypothesized cause and effect mechanisms explicated.

The lack of information about oral health may be compromising the use and quality of oral health care among racial/ethnic minority older adults. This finding is consistent with key informant views that senior center attendees did not regard oral health concerns with the same degree of immediacy as high blood pressure (indicative of hypertension) or high blood sugar (indicative of diabetes) [48]. Well-informed community members may fill critical roles in oral health promotion, as they are viewed as highly credible sources of information and recommendations to dental providers. Disseminating up-to-date information at frequented sites to older adults and the community at large about the importance of oral health, proper dental hygiene practices, and local oral health care options remain public health priorities.

\section{Additional file}

Additional file 1: Focused Group Interview Topic Guide. A topic guide consisting of open-ended questions that was developed by the research team and used by the focus group moderators to facilitate the sharing of personal experiences among participants regarding reasons why people may or may not visit a dentist. (PDF $57 \mathrm{~kb}$ )

\section{Abbreviations}

cmCause: Causal map cause; cmEffect: Causal map effect; cmID: Causal map identification number; dsCause: Data segment cause; dsEffect: Data segment effect; SegmentID: Data segment identification number

\section{Acknowledgments}

The authors thank the participants and recruitment staff whose engagement in this qualitative study made the reporting of the results possible.

\section{Funding}

The authors were supported in the research, analysis, and writing of this paper by the National Institute for Dental and Craniofacial Research (NIDCR) and the Office of Behavioral and Social Sciences Research (OBSSR) of the US National Institutes of Health $(\mathrm{NIH})$ for the project titled, "Integrating Social and Systems Science Approaches to Promote Oral Health Equity" (grant R01DE023072) and by the National Center for Advancing Translational Sciences (NCATS) of the NIH for the project titled, "Primary Care Screening by Dental Hygienists at Chairside: Developing and Evaluating an Electronic Tool" (grant UL1TR000038). The funding agencies had no role in the design of the study and collection, analysis, and interpretation of data or in the writing of the manuscript.

\section{Availability of data and materials}

De-identified raw data and materials described in the manuscript are freely available from the corresponding author on reasonable request.

\section{Authors' contributions}

SSK led the writing of this paper and conducted the analyses as part of her dissertation research. MEN closely edited the paper, provided interpretation, finalized the tables and figures, and contributed oral health and public health expertise. SSM conceived of and supervised the analyses, and contributed systems science expertise. All authors contributed to the writing and editing of this paper and approved it for publication.

Ethics approval and consent to participate

This research has been performed in accordance with the Declaration of Helsinki. The following Institutional Review Boards reviewed and approved all study procedures: Columbia University Medical Center Institutional Review Board [protocol AAAL4104(M01Y04)] and NYU School of Medicine Institutional Review Board (protocol i12-02947_CR4). All Health Insurance Portability and Accountability Act safeguards were followed. All participants signed written consent forms.

\section{Competing interests}

The authors declare that they have no competing interests.

\section{Publisher's Note}

Springer Nature remains neutral with regard to jurisdictional claims in published maps and institutional affiliations. 


\section{Author details}

'Department of Geography, The State University of New York at Buffalo, 115 Wilkeson Quad, Ellicott Complex, Buffalo, NY 14261-0055, USA. ${ }^{2}$ Department of Population Health, New York University School of Medicine, 227 East 30th Street, 8th Floor, New York, NY 10016, USA. ${ }^{3}$ Department of Epidemiology and Health Promotion, New York University College of Dentistry, 433 First Avenue, Room 726, New York, NY 10010, USA. ${ }^{4}$ Department of Sociomedical Sciences, Columbia University Mailman School of Public Health, 722 West 168th Street, New York, NY 10032, USA.

\section{Received: 10 April 2017 Accepted: 22 May 2018}

\section{Published online: 04 June 2018}

\section{References}

1. Centers for Disease Control and Prevention (CDC). Achievements in public health, 1900-1999. Fluoridation of drinking water to prevent dental caries. MMWR Morb Mortal Wkly Rep. 1999;48(41):933-40.

2. Centers for Disease Control and Prevention (CDC). Public health and aging: retention of natural teeth among older adults-United States, 2002. MMWR Morb Mortal Wkly Rep. 2003;52(50):1226-9.

3. Slade GD, Akinkugbe AA, Sanders AE. Projections of U.S. edentulism prevalence following 5 decades of decline. J Dent Res. 2014;93(10):959-65.

4. Kiyak HA, Reichmuth M. Barriers to and enablers of older adults' use of dental services. J Dent Educ. 2005;69(9):975-86.

5. Benjamin RM. Oral health: the silent epidemic. Public Health Rep. 2010; 125(2):158-9.

6. Fisher-Owens SA, Barker JC, Adams S, et al. Giving policy some teeth: routes to reducing disparities in oral health. Health Aff (Millwood). 2008; 27(2):404-12

7. Greenblatt AP, Estrada I, Schrimshaw EW, Metcalf SS, Kunzel C, Northridge ME. Acceptability of chairside screening for racial/ethnic minority older adults: a qualitative study. JDR Clin Trans Res. 2017;2(4):343-52.

8. Wu B, Liang J, Plassman BL, Remle RC, Luo X. Edentulism trends among middle-aged and older adults in the United States: comparison of five racial/ethnic groups. Community Dent Oral Epidemiol. 2012;40(2):145-53.

9. Shelley D, Russell S, Parikh NS, Fahs M. Ethnic disparities in self-reported oral health status and access to care among older adults in NYC. J Urban Health 2011;88(4):651-62.

10. Huang DL, Park M. Socioeconomic and racial/ethnic oral health disparities among US older adults: oral health quality of life and dentition. J Public Health Dent. 2015;75(2):85-92.

11. Northridge ME, Ue FV, Borrell LN, et al. Tooth loss and dental caries in community-dwelling older adults in northern Manhattan. Gerodontology. 2012:29(2):e464-73.

12. Penchansky $R$, Thomas JW. The concept of access: definition and relationship to consumer satisfaction. Med Care. 1981;19(2):127-40.

13. Powell M. On the outside looking in: medical geography, medical geographers and access to health care. Health Place. 1995;1(1):41-50.

14. Kim H, Andersen DF. Building confidence in causal maps generated from purposive text data: mapping transcripts of the Federal Reserve. Syst Dyn Rev. 2012;28(4):311-28

15. Phillips J, Ajrouch KJ, Hillcoat-Nallétamby S. Key concepts in social gerontology. Los Angeles: SAGE; 2010.

16. Sweeney K. Complexity in primary care: understanding its value. Oxford: Seattle: Radcliffe Pub.; 2006.

17. Greenhalgh T, Hurwitz B. Why study narrative? BMJ. 1999:318(7175):48-50

18. Northridge ME, Shedlin M, Schrimshaw EW, et al. Recruitment of racial/ ethnic minority older adults through community sites for focus group discussions. BMC Public Health. 2017;17(1):563.

19. Morgan DL. Focus group interviewing. In: Gubrium JF, Holstein JA, editors. Handbook of interview research: context and method. Thousand Oaks: Sage; 2002. p. 141-59.

20. Wenger GC. Interviewing older people. In: Gubrium JF, Holstein JA, editors. Handbook of interview research: context and method. Thousand Oaks: Sage; 2002. p. 259-78

21. Krueger RA, Casey MA. Focus groups: a practical guide for applied research. 4th ed. Thousand Oaks: Sage; 2009.

22. Paina $L$, Peters $D H$. Understanding pathways for scaling up health services through the lens of complex adaptive systems. Health Policy Plan. 2012; 27(5):365-73.
23. Ip EH, Rahmandad H, Shoham DA, et al. Reconciling statistical and systems science approaches to public health. Health Educ Behav. 2013:40(1 suppl):123S-31S

24. Metcalf SS, Northridge ME, Lamster IB. A systems perspective for dental health in older adults. Am J Public Health. 2011;101(10):1820-3.

25. McLeroy K. Thinking of systems. Am J Public Health. 2006;96(3):402.

26. Mabry PL, Marcus SE, Clark PI, Leischow SJ, Méndez D. Systems science: a revolution in public health policy research. Am J Public Health. 2010;100(7): 1161-3.

27. Luke DA, Stamatakis KA. Systems science methods in public health. Annu Rev Public Health. 2012;33:357-76.

28. Torrens PM. Geography and computational social science. GeoJournal. 2010; 75(2):133-48.

29. Carey G, Malbon E, Carey N, Joyce A, Crammond B, Carey A. Systems science and systems thinking for public health: a systematic review of the field. BMJ Open. 2015:5(12):e009002.

30. Ness RB, Koopman JS, Roberts MS. Causal system modeling in chronic disease epidemiology: a proposal. Ann Epidemiol. 2007:17(7):564-8.

31. O'Sullivan D, Evans T, Manson S, Metcalf S, Ligmann-Zielinska A, Bone C. Strategic directions for agent-based modeling: avoiding the YAAWN syndrome. J Land Use Sci. 2016:11(2):177-87.

32. Sterman J. Business dynamics: systems thinking and modeling for a complex world. Boston: Irwin/McGraw-Hill; 2000

33. Northridge ME, Metcalf SS. Enhancing implementation science by applying best principles of systems science. Health Res Policy Syst. 2016:14:74.

34. Black LJ. When visuals are boundary objects in system dynamics work. Syst Dyn Rev. 2013:29(2):70-86.

35. Ventana Systems, Inc. Vensim. Harvard; 2015

36. Ben-Shlomo Y, Kuh D. A life course approach to chronic disease epidemiology: conceptual models, empirical challenges and interdisciplinary perspectives. Int J Epidemiol. 2002;31(2):285-93.

37. Nicolau B, Thomson WM, Steele JG, Allison PJ. Life-course epidemiology: concepts and theoretical models and its relevance to chronic oral conditions. Community Dent Oral Epidemiol. 2007:35(4):241-9.

38. Shavers VL. Measurement of socioeconomic status in health disparities research. J Natl Med Assoc. 2007:99(9):1013-23.

39. Diez Roux AV. Complex systems thinking and current impasses in health disparities research. Am J Public Health. 2011:101(9):1627-34.

40. Noble J, Silverman E, Bijak J, et al. Linked lives: the utility of an agent-based approach to modeling partnership and household formation in the context of social care. In: Proceedings of the 2012 winter simulation conference; 2012. p. 1-12.

41. Salgado M, Marchione E, Gilbert N. Analysing differential school effectiveness through multilevel and agent-based modelling. J Artif Soc Soc Simul. 2014;17(4):3.

42. Metcalf SS, Northridge ME, Widener MJ, Chakraborty B, Marshall SE, Lamster IB. Modeling social dimensions of oral health among older adults in urban environments. Health Educ Behav. 2013;40(1 suppl):63S-73S.

43. Wang $\mathrm{H}$, Northridge $M E$, Kunzel $\mathrm{C}$, et al. Modeling social capital as dynamic networks to promote access to oral healthcare. Soc Cult Behav Model. 2016; 9708:117.

44. Chakraborty B, Widener MJ, Mirzaei Salehabadi S, et al. Estimating peer density effects on oral health for community-based older adults. BMC Oral Health. 2017;17(1):166.

45. Zhang Q, Northridge ME, Jin Z, Metcalf SS. Modeling accessibility of screening and treatment facilities for older adults using transportation networks. Appl Geogr. 2018;93:64-75.

46. Northridge ME, Kum SS, Chakraborty B, et al. Third places for health promotion with older adults: using the consolidated framework for implementation research to enhance program implementation and evaluation. J Urban Health. 2016;93(5):851-70.

47. Meadows DH. Thinking in Systems: A Primer. White River Junction: Chelsea Green Publishing Co.; 2008.

48. Marshall S, Schrimshaw EW, Metcalf SS, et al. Evidence from ElderSmile for diabetes and hypertension screening in oral health programs. J Calif Dent Assoc. 2015:43(7):379-87. 